\title{
Bose-Einstein Correlations in fully hadronic WW events at LEP2
}

\author{
Oliver Pooth* on behalf of the LEP/WW-FSI Working Group \\ EP Division, CERN, CH-1211 Geneva 23, Switzerland \\ E-mail: 'oliver.Pooth@ecern.ch'
}

ABstract: Bose-Einstein Correlations in WW events have been studied by all four LEP experiments. This overview concentrates on the results for the search for BoseEinstein Correlations between particles originating from different W's in the reaction $\mathrm{e}^{+} \mathrm{e}^{-} \rightarrow \mathrm{W}^{+} \mathrm{W}^{-} \rightarrow \mathrm{q} \overline{\mathrm{q}} \mathrm{q} \overline{\mathrm{q}}$, based on data recorded at centre-of-mass energies between 172 and $209 \mathrm{GeV}$. There is no evidence for Bose-Einstein Correlations between particles originating from different W's.

\section{Introduction to Bose-Einstein Correlations}

Bose-Einstein Correlations (BEC) between identical bosons are a well known phenomenon in high energy physics experiments and are often considered to be an equivalent of the Hanbury Brown \& Twiss effect in astronomy describing the interference of photons emitted incoherently. An alternative approach was proposed by B. Andersson et al. [i]1] taking into account a coherent particle production process in the framework of the Lund string model.

BEC manifests themselves as an enhancement of the production of identical bosons close in phase space and have been observed for two or more identical bosons in hadronic $\mathrm{Z}^{0}$ decays and single $\mathrm{W}$ decays (intra-W BEC) by all four LEP collaborations. The main interest at LEP2 is whether there is evidence for BEC between the particles coming from different W's (inter-WW BEC). In case of two hadronically decaying W's we have the unique situation of two partially overlapping hadronic systems [2]. The typical separation of the two $\mathrm{W}$ decay vertices is in the order of $0.1 \mathrm{fm}$ whilst the hadronization scale is in the order of $1 \mathrm{fm}$. Therefore in the incoherent scenario one expects similar correlations between particles originating from different W's as for particles originating from the same W. In the coherent scenario Bose-Einstein Correlations between the two systems may not exist at all. The two strings may decay independently as long as there is no color reconnection (see talk by D. Duchesneau, this proceedings).

${ }^{*}$ Speaker. 
A consequence of inter-WW BEC is a systematic uncertainty in the determination of the $\mathrm{W}$-mass in the $4 \mathrm{q}-$ channel. If inter-WW BEC affect particles from different W's a transfer of particles and/or momentum can disturb the $\mathrm{W}$ mass determination from the reconstructed invariant 2-jet masses. Initial predictions of various Monte Carlo models for $\Delta \mathrm{M}_{W}^{4 q}$ are in the range $0-100 \mathrm{MeV} / \mathrm{c}^{2}$. The latest LEP $\mathrm{W}$ mass combination quotes $\sigma\left[\mathrm{M}_{W}^{\mathrm{BEC}}(4 \mathrm{q})\right]=25 \mathrm{MeV} / \mathrm{c}^{2}$.

\section{Methods to study BEC}

BEC effects are usually studied in terms of 4-momentum differences $Q^{2}=-\left(p_{1}-p_{2}\right)^{2}$ and with the ratio of two-particle correlation functions $R\left(p_{1}, p_{2}\right)=\rho\left(p_{1}, p_{2}\right) / \rho_{0}\left(p_{1}, p_{2}\right)$ with $\rho\left(p_{1}, p_{2}\right)=1 / N_{e v} \cdot \mathrm{d} n_{\text {pairs }} / \mathrm{d} Q$. As a reference sample $\rho_{0}$ without BEC several possibilities can be used like e.g. unlike sign charged particle pairs, Monte Carlo samples without BEC or 'mixed' events where two hadronically decaying W's of independent semi-leptonic $\mathrm{WW} \rightarrow \mathrm{q} \overline{\mathrm{q}} \mathrm{l} \nu$ events are put together to fake a real $4 \mathrm{q}$ event that has no inter-WW BEC by construction.

\subsection{Access to inter-WW directly from data}

The following analysis method was proposed by Chekanov, De Wolf and Kittel [풀] used by all collaborations now. In the absence of inter-WW correlations and Poissonian multiplicity distributions the 2-particle density $\rho$ for like sign and unlike sign pairs can be written as

$$
\rho^{W W \rightarrow 4 q}=2 \cdot \rho^{W \rightarrow 2 q}+\rho^{W W_{m i x}}
$$

with $\rho^{W W \rightarrow 4 q}$ determined by the $4 \mathrm{q}$ sample, $\rho^{W \rightarrow 2 q}$ determined by the $\mathrm{q} \overline{\mathrm{q}} \mathrm{l} \nu$ sample after removing the leptonic part and $\rho^{W W_{m i x}}$ determined by events made of two independent $\mathrm{W} \rightarrow \mathrm{q} \overline{\mathrm{q}}$ events where only combinations of particles originating from different W's are used. Using relation 2.1 i sensitive test distributions can be set up to study inter-WW BEC:

$$
\begin{aligned}
\Delta \rho(Q) & =\rho^{W W \rightarrow 4 q}-2 \cdot \rho^{W \rightarrow 2 q}-\rho^{W W_{m i x}}, \\
D(Q) & =\frac{\rho^{W W \rightarrow 4 q}}{2 \cdot \rho^{W \rightarrow 2 q}+\rho^{W W_{\text {mix }}}} \\
D^{\prime}(Q) & =\frac{D(Q)}{D(Q)_{\mathrm{MC}, \mathrm{w} / \mathrm{o} \text { inter-WW BEC }}}
\end{aligned}
$$

If inter-WW BEC do not exist the test distributions will be $\Delta \rho(\mathrm{Q})=0$ and $\mathrm{D}(\mathrm{Q})=\mathrm{D}^{\prime}(\mathrm{Q})$ $=1$ for all $\mathrm{Q}$.

\subsection{Monte Carlo modeling}

The WW $\rightarrow$ 4q data sample contain 10-20\% background ${ }^{1}$, mainly $Z^{0 *} / \gamma \rightarrow 4$ jets. At this point all analyses rely on a correct Monte Carlo modeling since the background has to be subtracted with BEC MC samples.

\footnotetext{
${ }^{1}$ depending on the event selections of the experiment
} 
The MC implementation of BEC presently used in the analyses is a routine PYBOEI [4] that reshuffles particle momenta after the fragmentation to simulate the BEC effects, using a phenomenological parameterization

$$
R(Q) \sim 1+\lambda \cdot \exp \left(-r^{2} Q^{2}\right)
$$

with $\mathrm{r}$ being the source radius and $\lambda$ the BEC 'strength'. Various implementations can be tested, among them option 1: full (intra + inter) BEC, option 2: only intra-W BEC (no inter-WW BEC) or option 3: no BEC. All experiments tuned the PYBOEI/PYTHIA parameters $^{2}$ to the high statistics $Z^{0}$ data and checked the tune at high energies with $\mathrm{WW} \rightarrow \mathrm{q} \overline{\mathrm{q}} \nu$ events.

\section{Experimental results at LEP2}

The L3 collaboration uses the event mixing technique to obtain the reference sample with-


sign particle pairs. Data points are compared to scenarios with and without inter-WW

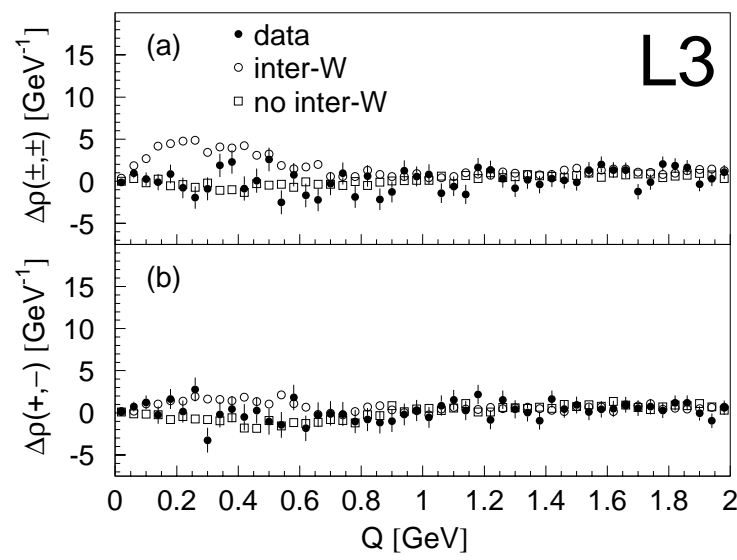

Figure 1: $\Delta \rho$ distribution (L3).
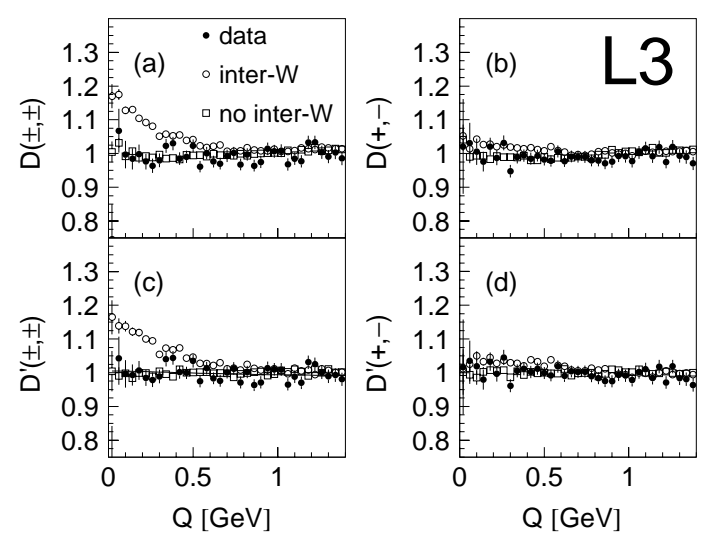

Figure 2: D and D' distributions (L3).

BEC. For the Monte Carlo model the inter-WW effect can be seen in an enhancement of like sign particle pairs in the low Q-region. Unlike sign particle pairs are artificially effected by the $\mathrm{MC}$ implementation due to the energy- and momentum conservation mechanism. The data ${ }^{3}$ prefers the 'no inter-WW BEC' scenario. To quantify the result an empirical fit to the D' distribution is performed using

$$
D^{\prime}(Q)=(1+\epsilon Q)\left(1+\Lambda \cdot \exp \left(-\sigma^{2} Q^{2}\right)\right) .
$$

The fit results for $\Lambda$ are $\Lambda^{\text {inter-WWBEC }}=0.126 \pm 0.008$ (stat.) and $\Lambda^{\text {data }}=0.008 \pm$ 0.018 (stat.) \pm 0.016 (syst.). L3 finds no evidence for inter-WW BEC disfavoring the interWW BEC MC with $4.7 \sigma$.

\footnotetext{
${ }^{2} \mathrm{QCD}$, fragmentation parameters and BEC parameters

${ }^{3}$ with background sutracted using a Monte Carlo sample
} 
In ALEPH a double ratio $\mathrm{R}^{*}$ of like sign and unlike sign particle pairs for data over

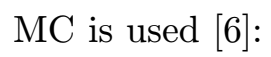

$$
R^{*}(Q)=\left(\frac{\rho^{++,--}(Q)}{\rho^{+-}(Q)}\right)^{\mathrm{data}} /\left(\frac{\rho^{++,--}(Q)}{\rho^{+-}(Q)}\right)_{\mathrm{w} / \mathrm{o} \mathrm{BEC}}^{\mathrm{MC}(\mathrm{WW}+\mathrm{q} \overline{\mathrm{q}}(\mathrm{bg}))}
$$

The analysis is based on the comparison of data with the different BEC MC scenarios. Figure ${ }_{-1}^{1} \bar{i}$ shows the double ratio $\mathrm{R}^{*}$. The analysis compares the $\mathrm{R}^{*}$ distribution determined

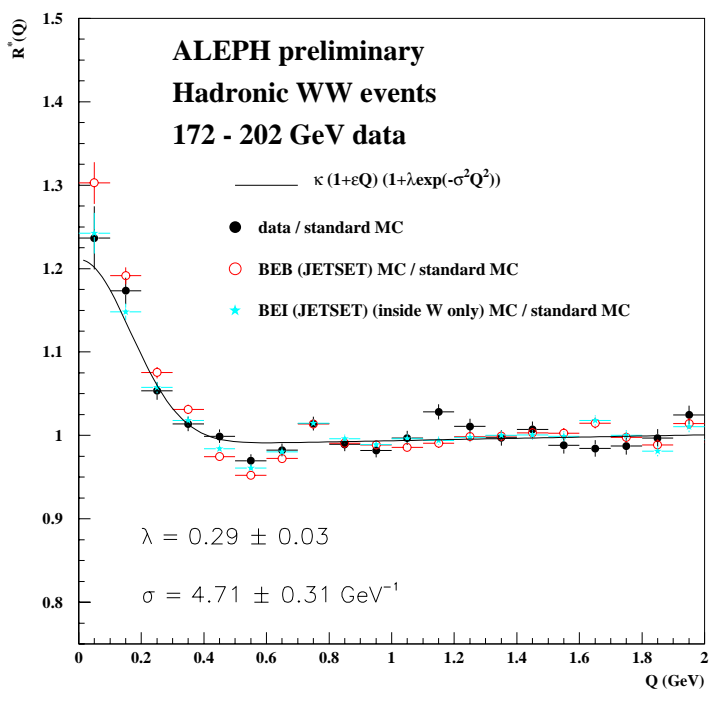

Figure 3: $\mathrm{R}^{*}$ distribution (ALEPH)



Figure 4: $\Delta \rho$ distribution (ALEPH)

from data with the different BEC MC scenarios (no inter-WW BEC (BEI) or inter-WW BEC (BEB)). Here the $Z^{0 *} / \gamma$ background is added to the WW sample. Fits are performed to the $\mathrm{R}^{*}$ distributions using a parameterization similar to Equation 2.5 .5 . The data is compatible with the intra-W BEC only scenario and disfavors the inter-WW scenario with $2.2 \sigma$. ALEPH also uses the mixed event technique with all data taken above $189 \mathrm{GeV}$ [i] In Figure 囱 the $\Delta \rho$ distribution is shown. The data prefers the no inter-WW BEC scenario.

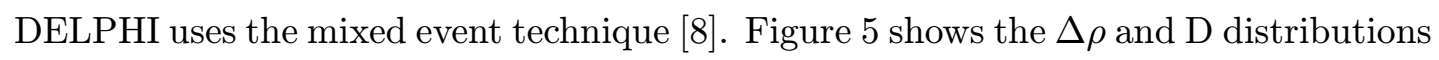
for like sign and unlike sign pairs. Fitting $D(Q)=N(1+\epsilon Q)(1+\Lambda \cdot \exp (-\sigma Q))$ leads to the results: $\Lambda^{\text {intra }+ \text { inter } \mathrm{BEC}}=0.24 \pm 0.03$ (stat.) and $\Lambda^{\text {data }}=-0.037 \pm 0.055$ (stat.) \pm 0.055 (syst.) for data ${ }^{4}$. DELPHI disfavors the inter-WW BEC MC with $3.2 \sigma$.

OPAL uses unlike sign particle pairs as reference without BEC [9i] and analyses the double ratio $\mathrm{R}(\mathrm{Q})$ : (data / $\mathrm{MC}$ without $\mathrm{BEC}$ ). $\mathrm{R}(\mathrm{Q})$ is studied for three samples at LEP2 energies enriched in $\mathrm{WW} \rightarrow \mathrm{q} \overline{\mathrm{q}} \overline{\mathrm{q}}, \mathrm{WW} \rightarrow \mathrm{q} \overline{\mathrm{q}} \nu$ and $\mathrm{Z}^{0 *} \rightarrow \mathrm{q} \overline{\mathrm{q}}$ events, whereas all three selected samples contain contributions from the others. Using MC information for the selection purities these samples are deconvoluted to get three contributions to extract

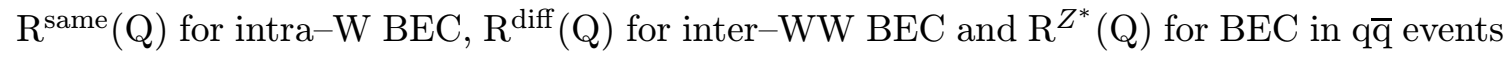

\footnotetext{
${ }^{4} \sigma$ is fixed to $1.01 \mathrm{fm}$, which is the fit result for the inter-WW BEC scenario
} 
by a simultaneous fit. In the absence of inter-WW BEC $\lambda^{\text {diff }}$ should be compatible with 0 . Figure $\overline{6}_{1}^{1}$ shows the OPAL distributions for the three samples. The results are $\lambda^{\text {same }}=0.69 \pm$ 0.12 (stat.) \pm 0.06 (syst.), $\lambda^{\mathrm{Z}^{*}}=0.43 \pm 0.06$ (stat.) \pm 0.08 (syst.) and $\lambda^{\text {diff }}=0.05 \pm 0.67$ (stat.) \pm 0.35 (syst.). Within the errors the data, taken between $172-189 \mathrm{GeV}$, is compatible with both no inter-WW BEC and inter-WW BEC. A publication using the mixing method and all recorded data is in preparation.

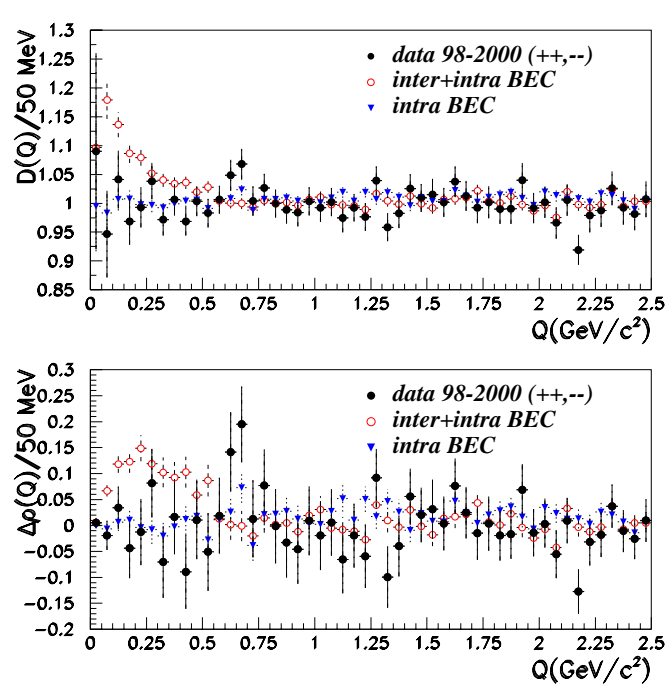

Figure 5: $\Delta \rho$ and D distributions (Delphi)

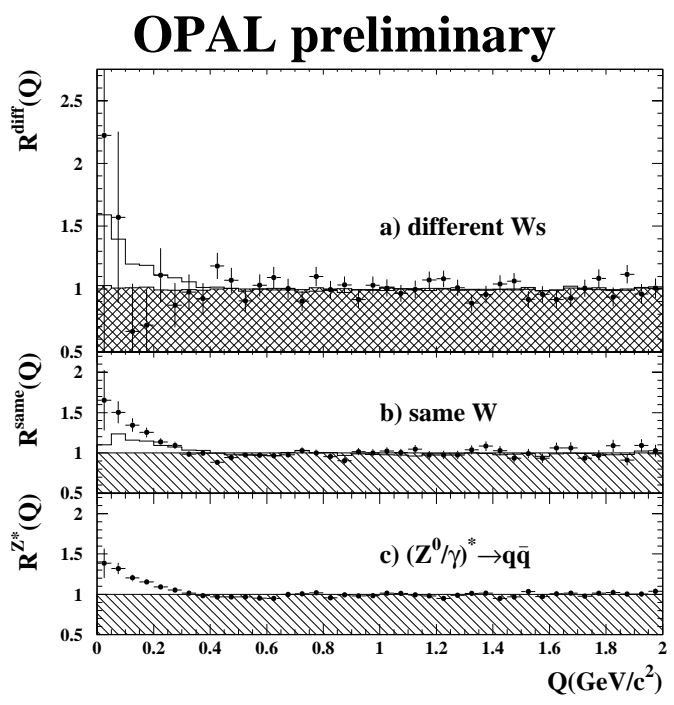

Figure 6: OPAL distributions

\section{Conclusion}

Using similar analysis techniques inter-WW BEC are not observed by the LEP experiments. BEC Monte Carlo models with the correlations between particles from different W's implemented in the same way as for the correlations inside one $\mathrm{W}$ cannot describe the data. For the future a combination of all data of the four collaborations using the mixing method is planned to achieve limits for inter-WW BEC using the combined data and to decrease the uncertainty in the $\mathrm{W}$ mass determination.

\section{References}

[1] B. Andersson and M. Ringnér,

[2] E. De Wolf, Correlations in $e^{+} e^{-} \rightarrow W^{+} W^{-}$hadronic decays, hep-ph/0101243.

[3] S. Chekanov, E. De Wolf, W. Kittel, 'Eur. Phys. J. C 2 (1999) 403.'.

[4] T. Sjöstrand, L. Lönnblad, Eur. Phys. J.

[5] L3 Note 2674, Bose-Einstein Correlations in $e^{+} e^{-} \rightarrow W^{+} W^{-}$Events at $\sqrt{s}=189-209 \mathrm{GeV}$, June $25,2001$.

[6] ALEPH collab., R. Barate et al., 'Ph hys. Lett. B $\mathbf{4} \overline{\mathbf{9}} \mathbf{3}(2000) 233$ 
[7] ALEPH 2001-064, CONF 2001-44, Further studies on Bose-Einstein correlations in W-pair decays, July 10, 2001.

[8] DELPHI 2001-107, CONF 534, Particle correlations in $e^{+} e^{-} \rightarrow W^{+} W^{-}$events with the DELPHI detector, May 15, 2001.

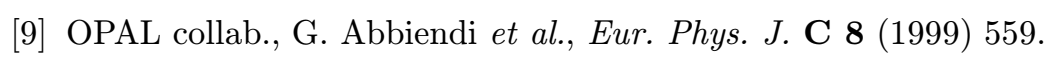

